\title{
CONOSCERE E CARTOGRAFARE L'IRAN, SPIE E CARTE DI IERI E DI OGGI. PIETRO DELLA VALLE E I VIAGGIATORI EUROPEI NELLA PERSIA DEL PRIMO ‘600 ALLA LUCE DEL PRESENTE
}

\author{
Raffaele Mauriello \\ (Allameh Tabataba'i University)
}

\begin{abstract}
ESTRATTO
Una delle caratteristiche della prima età moderna è lo sviluppo della cartografia. Fra il 1450 e il 1650 le carte passarono da rappresentare il mondo in modo medievale, in termini di orbis terrae, a una divisione del globo terrestre in segmenti formati da reticolati e coordinate.
\end{abstract}

Questo cambio fu dovuto in particolare ai numerosi viaggi di esplorazione o avventura e di natura diplomatica intrapresi in quegli anni dagli europei. Anche l'Italia vanta nomi importanti di viaggiatori che contribuirono a questo sviluppo. Nel caso dell'Iran, che all'epoca gli europei chiamavano Persia, il "nostro uomo" è Pietro della Valle (1586-1652). Lui c'era, e con lui lo sguardo e gli interessi dell'Italia, rappresentata all'epoca dallo Stato della Chiesa e dalla Repubblica di Venezia.

Ma della Valle non era solo. Con lui c'erano anche l'inglese Sir Robert Sherley, il tedesco Heinrich von Poser und Groß Naedlitz e lo spagnolo don García de Silva y Figueroa. Essi, ed altri che li seguirono, cercarono di informarsi ed influire sugli affari dell'Iran e della più ampia geopolitica del Vicino Oriente, contribuendo al contempo attraverso i loro resoconti di viaggio ed altri scritti a cartografare il paese.

Mutatis mutandis e in alcuni casi letteralmente sulle tracce dei loro predecessori, oggi come ieri $i$ viaggiatori europei continuano ad interessarsi del paese, e del suo rapporto con l'Europa.

\section{PAROLE CHIAVE}

Iran, Diplomazia, Cartografia, Intelligence, Seicento, Epoca Moderna, Pietro della Valle

\section{ABSTRACT}

One of the characteristics of the early Modern era is the development of cartography. Between 1450 and 1650 maps moved from portraying the world in Medieval terms - that is, as orbis terrae - to a division of the terrestrial globe in segments in the form of grids and coordinates.

This change was due in particular to the numerous travels of exploration or adventure and diplomacy undertaken in those years by Europeans. In the case of Italy and Iran, "our man" is Pietro della Valle (1586-1652). He was there, and with him the gaze and interests of Italy, represented at that time by the Vatican and by the Republic of Venice. But he was not alone.

Together with della Valle there were also the British Sir Robert Sherley, the German Heinrich von Poser und Groß Naedlitz and the Spanish Don Garcia de Silva y Figueroa. They, and others who followed them, tried to get information about and to influence the affairs of Iran and of the wider geopolitics of the Near East, at the same time making a contribution to the cartography of the country through their travel accounts and other writings.

Mutatis mutandis and in some cases literally following in the footsteps of their predecessors, today as yesterday European travelers continue to be interested in the country, and in her relationship with Europe.

\section{KEY-WORDS}

Iran, Diplomacy, Cartography, Intelligence, Seventeenth Century, Early Modern History, Pietro della Valle

Qui mores hominum multorum vidit, et urbes,

Ulisse 


\section{I viaggiatori europei del '600 e lo sviluppo della cartografia della Persia}

Una delle caratteristiche della prima età moderna è lo sviluppo della cartografia. Fra il 1450 e il 1650 le carte passarono da rappresentare il mondo in modo medievale, in termini di orbis terrae, a una divisione del globo terrestre in segmenti formati da reticolati e coordinate ${ }^{1}$. Ciò non solo permise una maggiore e migliore conoscenza del mondo, ma implicò un cambiamento nel modo stesso di guardare ad esso. Questo cambio fu dovuto in particolare ai numerosi viaggi di esplorazione o avventura e di natura diplomatica intrapresi in quegli anni dagli europei ${ }^{2}$. Come messo in luce da Bjola e Kornprobst (2018: 39), l'attività diplomatica ha una relazione di lunga durata - che va ben oltre i duemila anni di storia -, con quella di intelligence, e questo sembra essere il caso anche dei viaggiatoridiplomatici europei di cui è questione in questo articolo. All'epoca gli europei chiamavano 1'Iran 'Persia'. Il nome 'Iran', infatti, fa la sua comparsa nei trattati e nelle mappe prodotti in Europa solo a partire dagli inizi del XIX secolo, e all'inizio solo in forma limitata. Fino ad allora, il paese è denominato in prevalenza 'Persia', oltre che 'regno dei Sophi' (cioè dei Safavidi) a partire dal XVI secolo. Diversi paesi europei vantano nomi importanti di viaggiatori che contribuirono allo sviluppo della cartografia dell' $\operatorname{Iran}^{3}$. Nel caso dell'Italia, il "nostro uomo" si chiama Pietro della Valle (1586-1652). Lui c'era, e con lui lo sguardo e gli interessi dell'Italia, rappresentata all'epoca dallo Stato della Chiesa - mediatore più in generale degli interessi europei - e dalla Repubblica di Venezia ${ }^{4}$. Ma non era solo. Con lui c'erano anche l'inglese Sir Robert Sherley, che aveva preso il posto del fratello Anthony $^{5}$, il tedesco Heinrich von Poser und Groß Naedlitz e lo spagnolo don García de Silva y Figueroa ${ }^{6}$. Essi, ed altri che li seguirono, cercarono di informarsi ed influire sugli affari del paese e della più ampia geopolitica del Vicino Oriente, contribuendo al contempo attraverso i loro resoconti di viaggio ed altri scritti a cartografare l'Iran. Benché rinomato come paese di grande antichità e di rilevanza biblica, fino a circa il 1600 tanto la politica quanto la geografia della Persia erano rimaste in gran parte sconosciute agli europei. Le

\footnotetext{
${ }^{1}$ Questo periodo corrisponde in larga misura agli inizi dell'epoca moderna, quella che gli storici anglosassoni chiamano early modern period, epoca che, nel caso dell'Iran, corrisponde all'arco di tempo di vita dell'Impero safavide (1501-1722) e coincide in modo significativo e interessante con la nascita della modernità di matrice europea.

${ }^{2}$ Per una breve panoramica sulla Persia safavide attraverso lo sguardo dei viaggiatori europei si veda Matthee 2012. Per un quadro dei rapporti fra Europa e Iran a partire dalla seconda metà del Trecento fino all'epoca di nostro interesse, si veda Lockhart 1986, che mette in evidenza come il desiderio di un'azione militare concertata fra Oriente e Occidente contro i turchi ottomani, il cui crescente potere minacciava tanto la Persia come l'Impero bizantino e altri paesi europei, fosse al secondo posto tra le ragioni dei crescenti rapporti fra Persia ed Europa - le altre tre furono l'incentivo religioso, quello commerciale e il fatto che la Persia era terra di passaggio per i viaggiatori diretti o di ritorno dall'India e oltre.

${ }^{3}$ Va ricordato che, oltre ai viaggiatori e messi diplomatici, nel caso dell'Europa in Iran erano presenti all'epoca diverse missioni cristiane di agostiniani, carmelitani e cappuccini, stabiliti soprattutto a Isfahan; oltre a mercanti inglesi, olandesi e veneziani.

${ }^{4}$ E non è un caso, forse, che la sua opera sullo scià Abbas venne stampata proprio a Venezia, vista la forte censura vaticana.

${ }^{5} \mathrm{Nel}$ 1597, Sir Anthony fu per un periodo al servizio di scià Abbas I, prima di tornare in Europa, nel 1599, come membro di una missione diplomatica inviata dallo scià. Rimasto in Spagna, si mise al servizio del re Felipe III, per poi passare a quello di Felipe IV. Del suo resoconto di viaggio non si parlerà in questo articolo per motivi di spazio.

${ }^{6}$ Più in generale, fra il 1600 e il 1722, anno della caduta dei Safavidi, il numero dei visitatori europei è notevole. Oltre a quelli già menzionati, passarono per il paese raccogliendo informazioni: Cornelis Speelman, JeanBaptiste Tavernier, Raphaël du Mans, Jean de Thévenot, John Fryer, Jean Chardin, Engelbert Kaempfer, François Sansons, Cornelis de Bruyn e Artemii Volynskii, per non citare che i più importanti.
} 
principali notizie che arrivavano erano quelle di seconda mano scritte dai rappresentanti della Repubblica di Venezia stazionati a Costantinopoli, capitale dell'Impero ottomano.

La rinnovata volontà di conoscere il paese, tanto da un punto di vista geografico che politico, era anche dovuta alla prospettiva, avanzata da molti, di stabilire un'alleanza euroiraniana in chiave anti-ottomana ${ }^{7}$. Gli interessi erano compatibili. Gli europei-cristiani volevano "liberare" i luoghi santi della Palestina e soprattutto fermare l'avanzata turca nel Mediterraneo e nei Balcani; culminata, quest'ultima, nel fallito assedio di Vienna da parte degli ottomani del 1683. Gli iraniani, che nel 1501 con lo scià Isma'il (r. 1500-24) si erano ricostituiti in impero anche attraverso la scelta di fare dello shi'ismo la religione di Stato, erano anch'essi in lotta con la Sublime Porta $(B \bar{a} b-i$ ' $\bar{A} l \bar{l})$, con la quale confinavano in diversi punti dopo aver conquistato i territori della Mesopotamia e dell'Armenia. Il primo contatto avvenne nella decade del 1520, con lo scià Isma'il e l'imperatore Carlo I di Spagna (r. 15161556) e V del Sacro Impero Romano (r. 1520-1558). E l'idea fu presa in considerazione dai rispettivi successori, oltre che da diversi papi. In questo contesto, l'arrivo al potere in Iran di scià Abbas I (r. 1587-1629) diede un particolare impeto a questa dinamica. L'energica politica estera intrapresa da questi creò un ambiente politico ed economico che attrasse l'interesse di diversi attori europei rispetto al paese, visto come terra di opportunità religiose, commerciali e strategiche.

L'Iran di allora, come quello di oggi, veniva visto come fattore geopolitico potenzialmente determinante negli equilibri di potere su scala sia regionale che mondiale. Era quindi indispensabile accedere ad informazioni di prima mano sulle faccende di corte, oltre che conoscerne bene la geografia. Questo, di certo, era quello che pensava il nobile romano Pietro della Valle, personaggio che dà oggi il nome alla Scuola italiana di Teheran.

\section{Pietro della Valle ed il suo sguardo privilegiato sulle faccende di corte e sulla topografia dell'Iran}

Dico adunque che, avendo io fatto dimora sei anni nella Persia, e la maggior parte del tempo alla corte appresso di quel gran monarca, ricevuto et onorato da lui come ospite suo, con la continua prattica e con la diligente osservazione di tutte le sue cose, son venuto in conoscimento di sette condizioni di lui, degne al mio parere di molta lode ... Mostrerò che sia tale con le ragioni et esempi che appresso ne addurrò, contentandomi di sceglierne alcuni, anzi pochissimi, de' molti ch'io ne so, e che con gli occhi propri ne ho veduti. ${ }^{8}$

Correva l'anno 1616 e della Valle, che due anni prima era partito da Napoli per il pellegrinaggio in Terrasanta - passando da e soggiornando anche a Costantinopoli-, decideva di lasciare Aleppo, in Siria, vestito all'araba in direzione Bagdad per poi raggiungere l'Iran. Una volta giuntovi, in poco tempo il Nostro seppe farsi strada alla corte di Abbas I. Oltre che allo scià, della Valle ebbe accesso ai personaggi più influenti del paese e agli europei che in quegli anni passarono per l'Iran e che egli incontrò in particolare presso le residenze dello scià a Isfahan, Qazvin e Ashraf ol-Belad.

L'impareggiabile capacità di accesso alla corte di Persia da parte di della Valle fu favorita anche dalla spiccata capacità di apprendimento da parte del Nostro delle principali lingue islamiche del Vicino Oriente (arabo, persiano, turco e curdo) e, quindi, dalla possibilità

\footnotetext{
${ }^{7} \mathrm{Su}$ questo saliente aspetto della presenza missionaria europea nella Persia safavide, in particolare attraverso i gesuiti, si veda García Hernán 2015.

${ }^{8}$ Della Valle 2004: 33.
} 
di raccogliere informazioni "interne" sulla geografia politica, culturale e fisica dell'Impero safavide; otre che di quello ottomano e della Georgia. Nel suo diario personale e nelle lettere scritte durante i suoi viaggi, infatti, della Valle ci descrive una geografia dell'Iran che fa uso dell'approccio moderno dei cartografi dell'epoca, e cioè una topografia che utilizza in preferenza termini locali, invece di quelli della tradizione greco-latina, offrendo così ai cartografi di Venezia, Amsterdam, Antwerp e Parigi nuove e più utili informazioni per la mappatura del globo terrestre. Ancora più significativo risulta il suo De regionibus subiectis recentiori Persarum imperio ${ }^{9}$. Già la sola decisione di della Valle di descrivere l'Iran durante il regno di Abbas in un trattato di geografia è di per sé significativa. Come già menzionato, a marcare la differenza è soprattutto la scelta del Nostro di servirsi di preferenza di nomi e concetti geografici locali-islamici. In tal senso, va ricordato che le carte portolane prodotte a Genova e a Maiorca a partire dagli inizi del XIV secolo avevano già fatto degli italiani (e degli spagnoli, o meglio catalani) i primi a tracciare una più dettagliata geografia dell' Iran (e più in generale del Mar Mediterraneo e del Vicino Oriente fino alle sponde del Mar Caspio). E proprio il diario, le lettere e il trattato di geografia di della Valle marcano un importante passaggio nel processo di accumulazione di una conoscenza geografica capace di descrivere realtà correnti, superando il riferimento alla topografia del medioevo e dell'età classica. Questo fu possibile perché, andando oltre il riferimento alle informazioni reperibili nei libri tratti con lui dall'Italia, il Nostro preferì raccogliere informazioni e creare una conoscenza sui nomi di città, villaggi, fiumi, laghi, pianure, montagne e monumenti sulla base delle proprie osservazioni, informandosi presso cortigiani, studiosi, nobili, gente comune, contadini e nomadi. Ciò appare evidente soprattutto nelle lettere originali, mentre, nella versione pubblicata, della Valle bilanciò questo approccio facendo riferimento anche alle conoscenze geografiche rappresentate dalla tradizione classica. E questo bilanciamento lo ritroviamo nel De regionibus subiectis recentiori Persarum imperio, dove in ogni caso egli dà a entrambe le conoscenze (locale-islamica e classica greco-latina) uguale importanza; e, quindi, va di nuovo oltre l'uso dei racconti di viaggio del XVI e XVII secolo, che si limitavano a coprire le conoscenze orientali con l'abito di una prospettiva classica.

Un altro elemento di rilievo di questo lavoro fu lo sforzo di verificare e correggere gli errori presenti nei testi europei a sua disposizione, come il resoconto di viaggio del francese Pierre Bélon. A tal riguardo, quando lo ritenne necessario, della Valle effettuò escursioni per verificare che le informazioni locali, che contraddicevano quelle in suo possesso come europeo, fossero effettivamente corrette.

Insomma, dai numerosi scritti editi ed inediti lasciati da Pietro della Valle risulta evidente che questi intese il suo viaggio e la permanenza in Iran come finalizzati a raccogliere dettagliate informazioni tanto di natura politica quanto geografica.

\section{Della Valle e gli altri viaggiatori europei}

Ma in Iran della Valle non era solo. Il Nostro era a corte quando, nel 1619, l'inviato della corona spagnola (che dal 1580 al 1640 riunì anche quella portoghese) don García de Silva y Figueroa lasciava il paese dopo una permanenza di due anni, durante la quale aveva

\footnotetext{
${ }^{9}$ Il trattato all'epoca non venne pubblicato. Tuttavia, da una lettera inviata da della Valle al suo amico Mario Schipano e scritta dopo il suo ritorno a Roma nell'estate del 1626 sappiamo che Pietro in quell'anno aveva anche già scritto la dedicatoria del volume, la cui versione finale risale al 1628. Il manoscritto del testo originale è disponibile nella Biblioteca Estense di Modena in due versioni: quella preparata per la pubblicazione ( $\gamma . S .1 .10$, Fondo Campori 698) e una precedente, di difficile lettura ( $\gamma$. U.1.22, Fondo Campori 699). Il testo è stato pubblicato, in forma incompleta (assenza delle glosse e di alcune note, addizioni e cambi) e con diversi errori, a cura di Gamalero 1977: 287-303, col titolo "De recentiori imperio Persarum subiectis regionibus". Su questo aspetto dell'opera di della Valle, si vedano Brentjes e Schüller 2006: 169-219.
} 







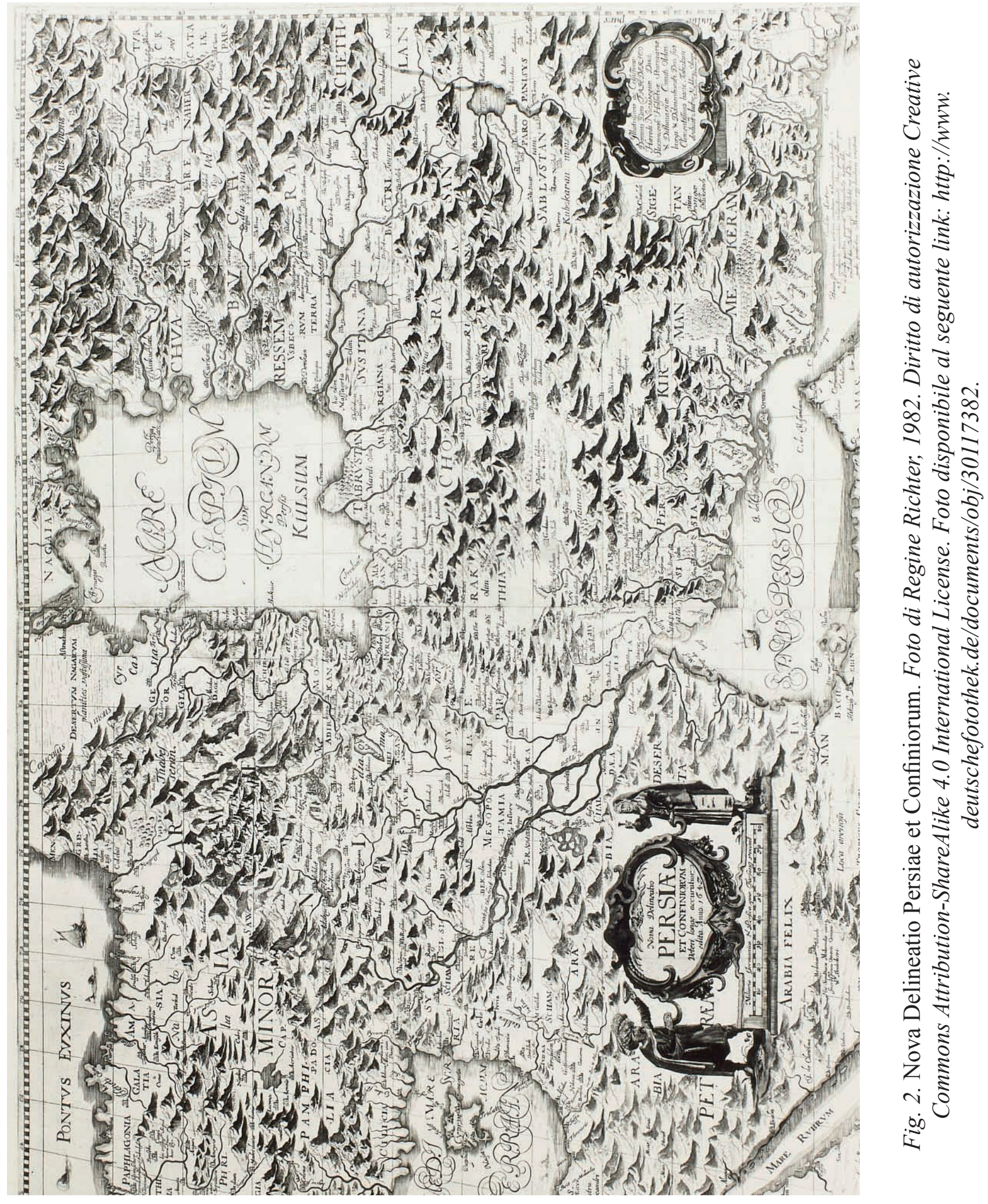


identificato, primo fra gli europei, l'ubicazione delle rovine dell'antica Persepoli, senza tuttavia essere riuscito a concludere gli importanti accordi di natura commerciale e politica che gli erano stati affidati. Un viaggio, quindi, di importanza cartografica, oltre che politica e commerciale. E ciò sembra essere confermato anche da un episodio decisamente più recente. È di un paio di anni fa, infatti, un viaggio attraverso l'Iran di Manolo Espaliú, fotografo sivigliano che, dotato di mezzi moderni come il GPS oltre che di una borsa di studio del Ministero degli Esteri dell'odierno Regno di Spagna, ha ripercorso, fotografato e cartografato il viaggio di duemila chilometri di don García dall'isola di Hormuz fino a Qazvin basandosi sulle informazioni fornite nel resoconto della sua missione diplomatica ${ }^{10}$.

Tornando alla missione diplomatica di Figueroa, essa non solo fallì, ma anzi, qualche anno dopo, nel 1622, l'Iran riprese il controllo dell'isola di Hormuz - con l'aiuto fondamentale delle navi della East India Company -, situata al centro dell'omonimo stretto all'entrata del golfo Persico e dal 1507 in mani portoghesi; i quali dal castello dell'isola da loro costruito nel 1515 ne controllavano tutta la navigazione. Va senza dire che la valenza geostrategica dell'isola non è molto cambiata. E anche quest'evento Pietro lo visse in prima persona, visto che era proprio sulla via di Hormuz, diretto in India, quando le forze di Abbas ripresero il possesso dell' isola togliendolo alla corona spagnola grazie all'aiuto di un altro stato europeo.

E della Valle era nei paraggi anche quando, nel giugno del 1621, un altro nobiluomo viaggiatore, Heinrich von Poser und Groß Nedlitz, visitando i giardini reali della capitale dell'impero, Isfahan, si imbatté nello scià Abbas. E fu proprio della Valle a guidarlo alla scoperta della città, come racconta lo stesso von Poser, meravigliato dalle conoscenze dell'italiano ${ }^{11}$.

Oltre a della Valle, in termini di cartografia dell'Iran del '600, importante risulta la figura di Adam Olearius (1599-1671), arrivato nel paese una decina di anni dopo il passaggio del Nostro ${ }^{12}$. Segretario e consigliere di Federico III, governatore del ducato di SchleswigHolstein-Gottorp in Germania, Olearius viaggiò in Iran nel quadro di una missione diplomatica in Russia e Persia finalizzata a stabilire una tratta alternativa per l'importazione della seta persiana nel nord Europa e di farlo in regime di monopolio. Avendo ottenuto il consenso dello zar, nel 1635 la missione si mise in marcia verso la capitale dell'Impero safavide, passando per Mosca, il Volga, Astrakhan e, dopo aver attraversato il Mar Caspio ed essere arrivata a Shamakhi (all'epoca in Iran, ma oggi parte della Repubblica dell'Azerbaijan), per Ardabil, Qazvin, Kashan e arrivando, infine, a Isfahan. Il dettagliato resoconto di viaggio di Olearius è ricco di mappe, fra cui risalta la Nova Delineatio Persiae et Confiniorum ${ }^{13}$.

Questo lavoro cartografico è di rilievo perché rappresenta il Mar Caspio in maniera molto più corretta delle carte barocche, in particolare rispetto alla sua maggior estensione da nord a sud rispetto a quella da est a ovest (si faccia il confronto, per esempio, con la carta Tabula Asiae VII di Sebastian Münster pubblicata nell'edizione della Geographia di Tolomeo del 1540).

\footnotetext{
${ }^{10}$ Il resoconto di viaggio di de Silva y Figueroa (1620) ha una storia piuttosto tormentata. In seguito alla morte di don García nel 1624 durante il viaggio di ritorno in patria, le tracce del manoscritto in gran parte si perdono. Nel 1668 esce a Parigi una traduzione parziale in francese di Abraham de Wicqfort. Nel 1852, Pascual de Gayangos trova per caso il manoscritto a Madrid presso un grossista di carta. Finalmente, fra il 1903 e il 1905 la Sociedad de Bibliófilos Españoles di Madrid pubblica la prima e unica edizione completa in spagnolo, in due volumi. Il testo è stato tradotto e pubblicato in inglese nel 2017 a cura di J. S. Turley e G. B. Souza, a testimonianza della sua rilevanza e attualità. Il lavoro di Espaliú è stato pubblicato nel 2018.

${ }^{11}$ Invernizzi, "Introduzione", in della Valle 2004: 23. Il dettagliato resoconto di viaggio di von Poser venne pubblicato nel 1675 .

${ }^{12}$ Il suo monumentale resoconto di viaggio in Russia e Iran venne pubblicato nel 1656. Per un'analisi di questo documento, si veda Brancaforte 2015.

${ }^{13}$ La mappa era già stata pubblicata, infatti, nel 1647, in un altro resoconto del suo viaggio intitolato Offt begehrte Beschreibung Der Newen Orientalischen Rejse.
} 


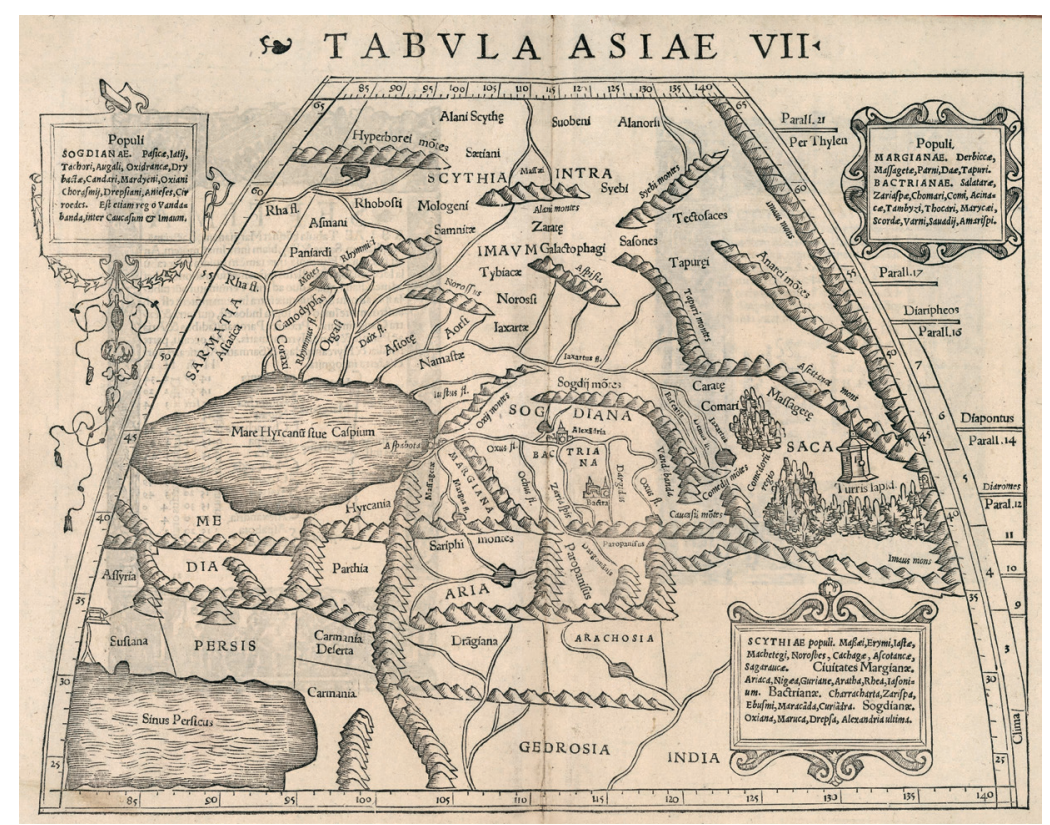

Fig. 3. Tabula Asiae VII tratta da Sebastian Münster, Geographia Universalis, Vetus et Nova, pubblicata da Henrichi Petri a Basel nel 1552. David Rumsey Map Collection. Foto disponibile al seguente link: https://www.davidrumsey.com/luna/ servlet/detail/RUMSEY 8 1 296545 90067926:Tabula-Asiae-VII?qvq=w4s:/ where\%2FRussia;lc:RUMSEY 8 1\&mi=349\&trs=1249\#.

Come nel caso di della Valle, la mappa dell'Iran di Olearius marca una transizione dall'era classico-medievale a quella rinascimentale nella conoscenza e rappresentazione geografica della Persia da parte degli europei.

\section{Carte e spie di ieri e di oggi. Pietro della Valle e i viaggiatori europei nella Persia del '600 alla luce del presente}

Nel caso dei personaggi descritti in questo articolo, a fare la differenza tanto rispetto alle informazioni sulla politica, società ed economica dell'Iran quanto a quelle di natura geografica da loro raccolte furono l'osservazione in prima persona e, nel caso dei più brillanti, la capacità di accedere a fonti orali e scritte in lingue islamiche. Questo perlomeno è il caso di Pietro della Valle, che fu in questo modo non solo capace di raccogliere informazioni più precise e dettagliate sulle faccende di Persia, ma anche di poterle interpretare lui stesso e presentarle secondo una prospettiva personale tanto alla Santa Sede quanto a un pubblico più generale senza dover passare attraverso lo sguardo, la mediazione e gli interessi di altri.

All'epoca descritta in questo articolo, tuttavia, l'alleanza euro-iraniana non si fece. Nel suo squisito ritratto di Abbas, della Valle racconta come lo scià fosse arrivato alla conclusione che la Spagna non era affidabile rispetto al possibile trattato di guerra contro l'Impero ottomano e che, inoltre, questa non rispettava gli accordi con l'Iran rispetto a Hormuz ${ }^{14}$. Mutatis mutandis, oggi è l'attuale leader del paese, Ali Khamenei, ad interrogarsi sulla effettiva volontà e capacità degli europei di rispettare gli accordi, e ci sono altri ad ascoltarlo e tradurne il pensiero sulla base di una conoscenza "interna" e di prima mano ${ }^{15}$.

\footnotetext{
${ }^{14}$ Della Valle 2004: 85.

${ }^{15}$ Marandi e Mauriello 2019: 18-38.
} 
Riguardo alla fallita alleanza, Invernizzi osserva che:

Gli interessi della Persia e della cristianità convergevano effettivamente, in toto o in parte. Eppure non si realizzò mai quel complesso organico di interventi da tanti auspicato, o per la difficoltà di coordinare le azioni di diversi protagonisti, che si muovevano invece spesso in tempi sfalsati pur perseguendo obiettivi comuni; o per la complessità della situazione che legava comunque in qualche modo al Turco gli interessi commerciali o politici di parte degli stati europei... ${ }^{16}$

A parte la considerazione che "al Turco" oggi si sono aggiunti alcuni nuovi attorni della regione, tocca ricordare a chi legge le parole dell'archeologo e storico dell'arte italiano che queste si riferiscono al Seicento, altrimenti qualcuno potrebbe (s)cambiarle per l'attualità.

Oltre all'alleanza militare, fallì anche il progetto dello scià Abbas di vendere direttamente agli europei la pregiata seta del paese evitando il transito e, quindi, i dazi attraverso 1'Impero ottomano. Mutatis mutandis, oggi sono altri ad avere interesse a che il commercio fra la Repubblica islamica dell'Iran e 1'Unione Europea non si faccia in maniera diretta - durante le precedenti sanzioni contro l'Iran una significativa parte dell'interscambio si faceva (e fa) attraverso gli Emirati Arabi Uniti e il petrolio veniva "girato" in parte alla Russia e da lì venduto al mercato europeo; con evidenti costi aggiuntivi tanto per gli iraniani come per gli europei - e non sfrutti tutto il suo importante potenziale.

Rimangono, tuttavia, i precedenti di natura diplomatica, che fanno a tutt'oggi di alcuni paesi europei partner preferenziali dell'Iran (Italia, Germania, Francia e Inghilterra in primis ma più di recente anche la Spagna), con un accesso privilegiato agli affari del paese. Così come rimasero le informazioni di natura geografica, che contribuirono a cartografare la Persia.

Oggi come nel Seicento, l'Iran e l'Europa sono in crescita e in cerca di un riposizionamento geopolitico dopo un periodo di ristrutturazione interna; nel primo caso attraverso una rivoluzione e la nascita della Repubblica islamica, nel secondo attraverso la creazione dell'Unione Europea. E oggi come allora gli interessi dell'Iran e dell'Europa convergono di nuovo, tanto in materia di sicurezza regionale e globale (lotta al terrorismo islamico e al traffico di droga, stabilizzazione del Vicino Oriente e dell'Afghanistan, controllo dei flussi migratori, etc.) quanto in termini di economia (energia e derivati in cambio di prodotti industriali e beni di consumo, coordinazione nella nuova Via della Seta, etc.).

Oggi, più che nel Seicento, l'Europa sembra essere riuscita a dotarsi di strumenti (come l'Alto Rappresentante per 1'Unione Europea per gli Affari Esteri e la Politica di Sicurezza) in grado di coordinare le azioni dei diversi protagonisti che la compongono, riducendo sensibilmente i movimenti di ognuno di essi in tempi sfalsati e perseguendo obiettivi comuni non solo impliciti ma dichiarati apertamente in campo internazionale.

Oggi, come allora, a fare la differenza sarà la capacità di una maggiore e diretta conoscenza reciproca, che sola potrebbe sanare gli errori di ieri. E oggi, come allora, potrebbe risultare determinante il nesso sapere-potere, rappresentato in modo paradigmatico da Pietro della Valle, uomo di conoscenza che tenta l'accordo (geo)politico.

\footnotetext{
${ }^{16}$ Invernizzi, “Introduzione”, in della Valle 2004: 14-15.
} 


\section{Bibliografia}

Bjola, C., Kornprobst, M., 2018, Understanding International Diplomacy: Theory, Practice and Ethics, London.

Brancaforte, E., 2015, “Mapping the Regnum Sophorum: Adam Olearius's Representation of the Safavid Empire (1647)", in W. Floor and E. Herzig (eds.), Iran and the World in the Safavid Age, London, pp. 293-308.

Brentjes, S., Schüller, V., 2006, "Pietro della Valle's Latin Geography of Safavid Iran (16241628): Introduction”, Journal of Early Modern History 10:3, pp. 169-219.

Espaliú, M., 2018, Viaje a Persia, Sevilla.

García Hernán, E., 2015, “The Holy See, the Spanish Monarchy and Safavid Persia in the Sixteenth Century: Some Aspects of the Involvement of the Society of Jesus", in W. Floor and E. Herzig (eds.), Iran and the World in the Safavid Age, London, pp. 181-203.

Lockhart, L., 1986, “European Contacts with Persia, 1350-1736”, in P. Jackson and L. Lockhart (eds.), The Cambridge History of Iran, vol. 6, The Timurid and Safavid Periods, London, pp. 373-409.

Marandi, S. M., Mauriello, R., 2019, “The Khamenei Doctrine: Iran's Leader on Diplomacy, Foreign Policy and International Relations", in N. Manabilang Adiong, D. Abdelkader and R. Mauriello (eds.), Islam in International Relations: Politics and Paradigms, London \& New York, pp. 18-38.

Matthee, R., 2012, "Safavid Iran through the Eyes of European Travelers", Harvard Library Bulletin 23: 1-2, pp. 10-24.

Olearius, A., 1656, Vermehrte Newe Beschreibung Der Muscowitischen vnd Persischen Reyse, So durch gelegenheit einer Holsteinischen Gesandschafft an den Russischen Zaar vnd König in Persien geschehen: Worinnen die gelegenheit derer Orter und Länder, durch welche die Reyse gangen, als Liffland, Rußland, Tartarien, Meden vnd Persien, sampt dero Einwohner Natur, Leben, Sitten, Hauß-Welt- vnd Geistlichen Stand mit fleiß auffgezeichnet, und mit vielen meist nach dem Leben gestelleten Figuren gezieret, zu befinden.

Rubiés, J.-P., 2011 (ed.), "Relación de la Embaxada que hiço en Persia Don Garçía de Silva y Figueroa (1620) by his secretary Saulisante", in R. M. Loureiro, A. C. Costa e V. Resende (eds.), Estudos sobre Don García de Silva y Figueroa e os "Comentários" de ambaixada à Pérsia (16141624), Lisboa, pp. 135-172.

Sherley, A., 1613, Sir Antony Sherley, His Relation of His Travels Into Persia, London.

de Silva y Figueroa, G., 1903-1905, Comentarios de la embajada que de parte del rey de España don Felipe III hizo al rey Xa Abas de Persia, M. Serrano y Sanz (ed.), Madrid.

Turley, J. S., Souza, G. B. (eds.), 2017, The Commentaries of D. Garcia de Silva y Figueroa on his Embassy to Shāh 'Abbās I of Persia on Behalf of Philp III, King of Spain, Leiden.

della Valle, P., 2004, Abbas re di Persia. Un patrizio romano alla corte dello Scià del primo '600, A. Invernizzi (ed.), Torino.

—, 1977, "De recentiori imperio Persarum subiectis regionibus", in Studi Iranici. Diciassette saggi di iranisti italiani, E. Gamalero (ed.), pp. 287-303.

—, 1843 Viaggi di Pietro della Valle Il Pellegrino Descritti da lui medesimo in Lettere Familiari all'Erudito suo Amico Mario Schipano Divisi in Tre Parti cioè: La Turchia, la Persia e l'India colla Vita e Ritratto dell'Autore, G. Gancia (ed.), Brighton. 
—, 1650, 1658, 1658, Viaggi di Pietro della Valle il Pellegrino Con minuto ragguaglio Di tutte le cose notabili osseruate in essi, Descritti da lui medesimo in 54. Lettere familiari, Da diuersi luoghi della intrapresa peregrinatione, Mandate in Napoli All'erudito, e fra' più cari, di molti anni suo Amico Mario Schipano, Diuisi in tre parti, cioè La Tvrchia, La Persia, e L'India, Le quali hauran per Aggiunta, Se Dio gli darà vita, la quarta Parte, Che conterrà le figure di molte cose memorabili, Sparse per tutta l'Opera, e la loro esplicatione, Roma.

—, 1628, Delle Conditioni di Abbàs Rè di Persia. All'Illustriss. \& Reuerendiss. Sig. Francesco Cardinal Barberino Nipote di N.S. Papa Vrbano VIII. Pietro della Valle il Pellegrino, Venezia.

von Poser und Groß Nedlitz, H., 1675, Der beeden Königl. ErbFürstenthūmer Schweidnitz und Jauer in Schlesien Hochverordneten LandesBestllten des HochEdelgebohrnen Herren Heinrich von Poser und Groß Nedlitz, Lebens und TodesGeschichte. Worinnen das TageBuch seiner Reise von Costantinopel aus durch die Bulgarey, Armenien, Persien und Indien ans Liecht gestllet von Dessen danckbahrem Sohbne Heinrich von Poser und Grob Nedlitz. 